\title{
Potencial de inundación de la quebrada el Monte (hidráulica), Bolivia
}

\author{
Flood potential of the El Monte stream (hydraulic), Bolivia
}

\author{
Eynar Sánchez Yelma \\ eynarsy@gmail.com \\ Universidad Católica Boliviana “San Pablo", Bolivia \\ Kevin Alex Cardozo Herrera \\ wincho12345@gmail.com
}

Artículo recibido enero 2019 | Arbitrado en feb-marzo 2019 | Publicado en mayo 2019

\section{RESUMEN}

En los últimos años el ordenamiento territorial, es sin duda, uno de los mayores problemas que enfrenta el Departamento de Tarija, producto de su crecimiento y desarrollo, ha causado que el crecimiento hacia las zonas naturales, ocasionando cambio en el uso de suelo, lo que ha derivado que muchas construcciones se encuentren en las zonas más vulnerables de sufrir un daño debido a un desastre natural, como es el caso de las inundaciones. La investigación se divide en tres estudios: estudio hidrológico, estudio hidráulico y elaboración de mapas de inundación. El estudio tiene como objetivo la elaboración de mapas de inundación de la Quebrada El Monte en el tramo que abarca desde el Hospital Obrero (Calle Rosendo Estensoro) hasta la unión con la Quebrada San Pedro, abarcando una longitud de 2870 metros; realizando las respectivas evaluaciones hidrológicas e hidráulicas, mediante los programas HEC-HMS y HEC-RAS, para así determinar los lugares con mayor peligro de inundación en la parte urbana con el fin de prevenir daños significativos en la población. Se determinó que debe ser estudiado de manera puntual las zonas críticas, ayudando con ello, al desarrollo del departamento de Tarija en la zona urbana.

Palabras clave: Inundación; ordenamiento territorial, quebrada, zonas en riesgos

\begin{abstract}
In recent years, land use planning is undoubtedly one of the biggest problems facing the Department of Tarija, as a result of its growth and development, it has caused growth towards natural areas, causing changes in land use, which This has resulted in many buildings being in the most vulnerable areas to suffer damage due to a natural disaster, such as floods. The research is divided into three studies: hydrological study, hydraulic study, and flood mapping. The objective of the study is to prepare flood maps for the Quebrada El Monte in the section that runs from Hospital Obrero (Calle Rosendo Estensoro) to the junction with Quebrada San Pedro, covering a length of 2870 meters; carrying out the respective hydrological and hydraulic evaluations, through the HECHMS and HEC-RAS programs, in order to determine the places with the greatest risk of flooding in the urban part in order to prevent significant damage to the population. It was determined that critical areas should be studied in a timely manner, thereby helping the development of the department of Tarija in the urban area.
\end{abstract}

Key words: Flood; territorial ordering, ravine, areas at risk 


\section{INTRODUCCIÓN}

La hidráulica estudia detalladamente cada uno de los eventos relacionados con el flujo de caudal de la red hidrográfica de una cuenca, siendo uno de los principales objetivos de estos estudios determinar qué riesgos pueden ocasionar.

Las avenidas o crecidas extremas son uno de los fenómenos hidrológicos que requieren de estudio, debido a que este tipo de eventos se caracterizan por ocasionar daños a la variación extrema de caudales poniendo en riesgo vidas humanas $\mathrm{y}$ pérdidas materiales. La provincia Cercado municipio del departamento de Tarija en Bolivia forma parte de la cuenca del Río Guadalquivir dividida en diferentes sub cuencas las cuales ha sido objeto de diferentes estudios de índole ambiental, debido al crecimiento no controlado de la ciudad, el cual ha causado que muchas obras civiles estén construidas a las orillas de los ríos. Las quebradas con más presencia urbana en la ciudad de Cercado son la El Monte y de San Pedro, así convirtiéndose en una prioridad el estudio de las cuencas de dichas quebradas. Históricamente la ciudad de Tarija ha sido afectada en diversas ocasiones por desastres naturales causados por el Rio Guadalquivir y su afluente como la quebrada de El Monte es por ello que resulta necesario realizar un estudio para determinar las zonas con mayor riesgo, especialmente por inundación, debido a que la mancha urbana que está muy próxima a esta.

La simulación por computador con el uso de software como el HEC-RAS es una alternativa viable para realizar un estudio de este tipo y con ayuda de un levantamiento topográfico brindan resultados confiables.

El estudio se enfoca en realizar modelación hidráulica en la sub cuenca de la
Quebrada El Monte con el fin de determinar la vulnerabilidad de los terrenos y obras civiles próxima; para la realización de dichas simulaciones se contó con información, mapas, eventos históricos, entre otros, provenientes de instituciones públicas $\mathrm{y}$ privadas.

La investigación de largo alcance ayudará al desarrollo del departamento de Tarija tanto en la zona urbana como en la rural. El departamento de Tarija está ubicado al sur de Bolivia en la frontera con las repúblicas de Argentina y Paraguay. Está situado entre los paralelos $20^{\circ} 53^{\prime} 00^{\prime \prime}$ y $22^{\circ}$ $52^{\prime} 30^{\prime \prime}$ de Latitud Sud y entre los meridianos

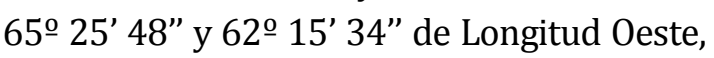
con características geográficas variadas y relieves que van desde los $4.000 \mathrm{msnm}$ hasta los $300 \mathrm{msnm}$. La Provincia Cercado, se encuentra situada en el corazón del departamento de Tarija, entre los paralelos $21^{\circ} 00^{\prime}$ y $22^{\circ}$ 50' de latitud sud y los meridianos $62^{\circ} 1^{\prime} 5^{\prime}$ y $65^{\circ} 20^{\prime}$ de longitud Oeste de la Línea de Greenwich

\section{Proceso de expansión}

La ciudad de Tarija se ha visto afectada notablemente por el crecimiento urbano. La expansión espacial de la ciudad se ha desarrollado de forma desordenada y poco planificada, impulsada principalmente por el crecimiento demográfico en la ciudad. La superficie de los barrios de La Pampa, Virgen de Fátima, El Tejar, Aniceto Arce, Juan XXIII y San Gerónimo muy próximos a la Quebrada El Monte, en los últimos años casi ha duplicado sus áreas construidas y han incluido el cambio substancial de los usos y coberturas de los suelos. Superficies de suelos con coberturas naturales como bosques, lechos de ríos y quebradas han sido urbanizadas, perturbando severamente los flujos de materia y energía al interior de 
las cuencas donde se localizan estos barrios. En particular el uso de la tierra y el cambio de gestión de la tierra afectan a la hidrología disminuyendo los recursos hídricos e incrementando el riesgo de inundación.

\section{Impactos de la urbanización en el sistema hídrico}

La impermeabilización de los suelos que se concreta con la expansión de la ciudad afecta directamente a la cuenca presentando una interferencia en el ciclo hidrológico donde el asfalto y el concreto reemplazan al suelo, desagües y conductos de agua. El impacto de la expansión sobre el sistema hidrológico es el aumento de escorrentía superficial aumentando así el caudal y la velocidad. También se considera el incremento de pendiente de cursos de agua aumentando la escorrentía incrementando el flujo de aguas perdiendo su capacidad de infiltración. Estos aumentos de flujo contribuyen a las inundaciones directamente haciendo difícil la evacuación de estos creando una posible inundación.

\section{Riesgo de inundación}

Para la conceptualización de riesgo se consideran dos factores importantes; la amenaza o peligro y la vulnerabilidad. El peligro es como la magnitud y duración de una fuerza potencial peligrosa por su capacidad de destruir un ecosistema. La amenaza es la reducida capacidad del sistema para adaptarse a determinadas circunstancias; esto depende del grado de exposición, protección, reacción inmediata; la vulnerabilidad es difícil de cuantificar; se puede reducir mediante obras hidráulicas de protección y la planificación de territorio. El impacto de riesgo de inundación en zonas urbanas es muy alto porque se ven incrementadas por el impacto de actividad humana.

\section{Mitigación de riesgos}

Los mapas de riesgos por inundación y el análisis de vulnerabilidad para diferentes áreas son herramientas útiles para la planificación futura de la urbanización. Las decisiones sobre dónde construir casas, fábricas, obras hidráulicas de protección para la urbanización que son reconocidas como herramientas claves en la gestión de los riesgos de inundaciones futuras son difíciles de tomar porque no existe información que pueda indicar el riesgo potencial en diferentes áreas de la ciudad.

En el plan de ordenamiento municipal de Tarija que promueve alcanzar la calidad de vida y el vivir bien no hace referencia a ningún área con riesgo de inundación. Tal que debería abarcar la identificación, organización y regulación de las actividades humanas viendo el comportamiento de cada elemento del sistema.

Para lo que este estudio estuvo enfocado en la realización de las simulaciones hidrológicas e hidráulicas mostrando el potencial a consecuencia de crecidas en la cuenca generando así mapas de inundación.

Actualmente se tiene información escasa acerca de las zonas de inundación; es una de las razones por las que existe un mal ordenamiento territorial en el cual se encuentran construcciones civiles, manejo económico y social dentro de posibles áreas de inundación. Por ejemplo, el Hospital Obrero, Zona de restaurante Av. Los Membrillos, Universidad Autónoma Juan Misael Saracho, Club tenis Tarija. Para ello, se plantea la interrogante acerca de que si $¿$ Es posible generar simulaciones de inundación con el uso de conocimientos de hidrología e hidráulica aplicados a software 
que muestren los potenciales riesgos de crecidas de la Quebrada El Monte que nos ayuden a generar mapas de inundación para crear planes de emergencia para condiciones extremas y regular el crecimiento de la ciudad?

Marcando de esta manera el objetivo o propósito de la investigación el cual es elaborar modelación hidráulica de la Quebrada El Monte, con ayuda del programa HEC- RAS utilizando datos de caudales para diferentes periodos de retorno.

La importancias de este estudio está basada en el incremento en la vulnerabilidad de la ciudad de Tarija ante la presencia de fenómenos naturales cada vez más intensos y frecuentes, que conllevan a la necesidad de formular estrategias para introducir medidas apropiadas de prevención y mitigación en base a un análisis de vulnerabilidad, haciendo de vital importancia que se cuente con instrumentos de planeación ante estos eventos.

Los modelos hidráulicos son de gran uso para la realización de mapas de inundación como principal instrumento en Tarija; son escasos o pocos accesibles a ellos haciendo el uso de zonas de inundación para la construcción de edificaciones, caminos; siendo un problema para el ordenamiento territorial.

El presente artículo se fundamenta en el sentido de servir como una herramienta preventiva y de apoyo para las autoridades en la toma de decisiones ante la incertidumbre asimismo pretende servir como medio de información para la población.

\section{METODOLOGÍA}

Se emprendió el estudio de la investigación con la recopilación de información, datos proporcionados por el
SENAMI, herramientas necesarias y disponibles en los municipios que abarca la Quebrada El Monte como ser: Programa Google Earth Pro; Programa ArcGIS; Programa HEC-HMS, y Programa HEC-RAS.

Análisis Hidrológico. Esta etapa se subdivide en tres partes características de la cuenca, análisis pluviométrico e ingreso de datos al programa HEC- HMS los cuales nos ayudó a obtener el flujo máximo que se genera en el punto de aforo.

Análisis Hidráulico. Esta etapa se subdivide en tres partes levantamiento topográfico de la zona de estudio, ubicación de secciones e ingreso de datos al programa HEC-RAS.

Elaboración de Mapas de Inundación. Esta etapa se subdivide en dos exportaciones de puntos topográficos al programa Arc GIS.

Se encontró estudios relacionados con el análisis hidrológico que se realizaron en zonas específicas de la Quebrada el Monte, los cuales se mencionaran a lo largo del artículo, estos son: Control de Inundaciones de la Ciudad de Tarija PEA (1999); Estudio de Avenidas Máximas para Prevenir Inundaciones del Río Pilcomayo en la Ciudad de Villa Montes. Ribstein, y Pena, (1993 citado por AA 2014); Evaluación del riesgo de inundación de la Quebrada el Monte de la ciudad de Tarija por Terrazas (2011), Estudio del riesgo de inundación en la quebrada de San Pedro en la ciudad de Tarija de Olaguivel (1999).

\section{RESULTADOS}

Para el inicio del proceso investigativo se puso en consideración la magnitud de las inundaciones en los últimos años en el 
departamento de Tarija; al no contar con estudios previos que puedan exponer zonas con riesgo de inundación, se tomó la decisión de realizar una investigación de la cuenca de la Quebrada El Monte cual pasa por el centro de la ciudad de Tarija.

Se emprendió el estudio de la investigación con la recopilación de información y herramientas necesarias y disponibles en los municipios que abarca la cuenca de la Quebrada El Monte instituciones encargadas, como son: Cartas Geográficas del Instituto Geográfico Militar (IGM); Programa Google Earth Pro;
Programa ArcGIS; Ubicación de Estaciones Pluviométricas; Datos de Estaciones Pluviométricas (Proporcionadas por el SENAMHI), y el levantamiento topográfico de la zona de estudio. Para la modelación hidráulica se utilizó HEC-RAS.

\section{Análisis hidráulico HEC-RAS}

Luego de haber obtenido los caudales máximos diarios mediante el software HEC-HMS proporcionados por la tesis "investigación del potencial de inundación de la quebrada el monte (hidrología)".

Tabla 1. Caudales de diseño

\begin{tabular}{cc}
\hline PERÍODO DE RETORNO & CAUDAL DE DISEÑo $\left[\mathbf{m}^{\mathbf{3}} / \mathbf{s}\right]$ \\
\hline $\mathbf{2}$ años & 39.6 \\
$\mathbf{5}$ años & 54.2 \\
$\mathbf{1 0}$ años & 37.3 \\
$\mathbf{2 5}$ años & 84.4 \\
$\mathbf{5 0}$ años & 97.5 \\
$\mathbf{1 0 0}$ años & 110.7 \\
$\mathbf{3 0 0}$ años & 131.8 \\
$\mathbf{5 0 0}$ años & 141.7 \\
\hline
\end{tabular}

La siguiente inquietud es conocer cómo recorre la cuenca el curso de agua. Se simula este proceso con el empleo del software HEC-RAS para el correspondiente análisis hidráulico de la cuenca de la Quebrada El Monte.

Para empezar la modelización hidráulica de la cuenca es necesario seguir tres etapas fundamentales; la primera consiste en el levantamiento topográfico de la zona de estudio, la segunda consiste con la ayuda del Civil 3D generar los datos complementarios para su posterior exportación al HEC-RAS, y la tercera es la exportación y generación de los resultados para la generación de modelos hidráulicos.

\section{Inspección de campo}

Para la identificación de puntos o zonas críticas de la Quebrada EL Monte así como para evaluar el estado actual del cauce y los márgenes se partió de:

- Análisis de imágenes de Google Earth, donde se señalaron algunas posibles zonas de riesgo de inundación.

- Recorrido a lo largo del tramo de estudio. 
El recorrido permitió definir puntos o zonas a estudiar desde el punto de vista topográfico e hidráulico.

\section{Basura en el Quebrada}

Dando el recorrido por el tramo de estudio se pudo advertir con pesar la contaminación debido hacinamiento de la basura tanto en el lecho de la Quebrada como en sus márgenes. Apoyando este punto de vista se muestra a continuación una información dada por los periódicos locales "EL País" y "El Nacional".

\section{Las quebradas se convirtieron en basureros y cementerios de chatarra}

Las quebradas que tiene la ciudad de Tarija están cada día más contaminadas. En un recorrido realizado por EL NACIONAL, (nov-2010) se constató que hay más basura y chatarra que agua. Se puede encontrar todo tipo de materiales, desde llantas, planchas, mesas de plástico, bicicletas, latas, pedazos de televisores, radios, equipos eléctricos, juguetes, balones, entre otros.

Los puntos más críticos son las quebradas que desembocan a la Víbora Negra y están detrás del Mercado Campesino. En ese lugar hay alcantarillas que convergen en los principales afluentes de la capital Chapaca, lo cual genera una mala imagen turística porque las pocas áreas verdes están contaminadas y son auténticos basurales.

Debido a que todavía no llovió en la ciudad de Tarija, estas quebradas están secas. En todo caso, cuando llegue el gran caudal, la basura acumulada será arrastrada por la quebrada de la Víbora Negra hasta la quebrada del Monte, y finalmente desembocara en el río Guadalquivir.
Ante este panorama, algunos ambientalistas sugieren realizar una campaña de limpieza en estas quebradas antes que comience la temporada de lluvias, porque el gran afectado será el principal afluente de la ciudad, que se constituye en el patrimonio Chapaco.

En este recorrido también participó el presidente de la Sociedad Protectora de Animales de Tarija (SPAT), Gonzalo Torrez, quien lamentó que las autoridades no hagan nada para dar una solución definitiva al problema del medio ambiente, y en especial a la contaminación de quebradas y ríos.

Asimismo, hizo notar que no existen carteles con leyendas para concientizar a la gente que vive en los alrededores de las quebradas a fin de que no boten su basura en este lugar. Tampoco hay efectivos de la Policía Urbana o funcionarios de la Unidad de Medio Ambiente para realizar un trabajo de control.

El activista recordó que estas quebradas serán embovedadas y para lo único que servirán será para tapar todo el desastre que se hace con los afluentes de Tarija, ya que la basura y los desagües circularan por debajo, cuando en realidad lo que se debe hacer es preservar las áreas verdes con sus árboles alrededor.

Asimismo, Torrez convocó al Comité Cívico para que promueva la preservación y la limpieza de la quebrada de Tarija, porque entre sus muchas funciones está el velar por el patrimonio Chapaco, cosa que a la fecha no lo hizo.

También pidió a la Entidad Municipal de Aseo de Tarija (EMAT) para que tome en cuenta, dentro de su campaña de limpieza, las quebradas que requieren cuanto antes un tratamiento especial antes que llueva y arrastre toda la basura al río Guadalquivir. 
"No es posible que las autoridades no hagan nada para preservar el medio ambiente. Yo realizaré acciones legales porque cada día se encuentra más atrocidades en contra de la naturaleza. El hecho de embovedar las quebradas no cumple con sus objetivos porque continuará la contaminación, seguirá siendo un lugar inseguro y los malos olores molestarán a los vecinos", comento.

"El hecho de embovedar las quebradas no cumple con sus objetivos porque continuará la contaminación, seguirá siendo un lugar inseguro y los malos olores molestarán a los vecinos".

\section{Hay quejas por basura $y$ malos olores de la quebrada El Monte}

La quebrada El Monte actualmente es un depósito de basura doméstica y aguas servidas, alrededor existen casas y está el Hospital Obrero. Esta situación preocupa porque puede agravarse y causar daños a la salud. (Gutiérrez, El País, Oct 18, 2014).

Esos desechos, más aún durante épocas de calor, se descomponen y generan olores desagradables. La Cooperativa de Servicios de Agua y Alcantarillado de Tarija (Cossalt) se comprometió a inspeccionar el lugar para conocer de dónde vienen las aguas que desembocan en esa quebrada.

\section{Vecinos preocupados}

Los vecinos se quejan de que las personas de los alrededores echan su basura a la quebrada, incluso perros muertos. La señora Martha Choque dijo que constantemente botan, en ese lugar, todo tipo de desechos. "No se puede vivir así, las autoridades que correspondan deben limpiar está quebrada", expresó.
Otro vecino, Manuel Flores manifestó que la quebrada se ha vuelto un baño público, "se agrava más la situación cuando habilitan ferias por esta zona".

María Méndez, preocupada, señaló que esa contaminación de la quebrada puede ocasionar que se propaguen enfermedades. "A la gente de alrededor se le ha hecho costumbre botar sus basuras inconscientemente. Espero que las autoridades puedan controlar esta situación y hacer la limpieza".

Preocupados, los vecinos pidieron a las autoridades de la Alcaldía limpiar la quebrada El Monte, porque el calor puede agravar la situación.

\section{Alcantarillado}

Otro factor que afecta a los vecinos son las aguas servidas que desembocan hacia la quebrada el Monte de la boca de tormenta que existe en el lugar, que al parecer son aguas pluviales. "Hay evidentemente otras aguas, que inclusive salen a la altura del Hospital Obrero, de otras cámaras, pero es agua pluvial", explicó el gerente de Cosaalt, Benito Castillo.

Aclaró que las aguas de los hospitales cuentan con el sistema de alcantarillado sanitario de la Cooperativa. "Nosotros hacemos el traslado correspondiente a las lagunas de oxidación", hay evidentemente otras aguas que salen a la altura del hospital de otras cámaras, pero es agua fluvial.

Sin embargo, la autoridad se comprometió a inspeccionar el lugar para conocer qué tipo de aguas desembocan en esta quebrada. "Nosotros podemos ir a inspeccionar, yo puedo mandar gente el día lunes en el lugar para que verifique y pedir un informe técnico", dijo. Explicó que los desechos que botan de los hospitales y de las casas van directamente al alcantarillado 
sanitario de la empresa, pero no se puede hacer un tratamiento de las aguas servidas porque al alcantarillado llegan bolsas plásticas, trapos y todo tipo de residuos.

Indicó que la planta de tratamiento se hace exclusivamente para aguas servidas domésticas, no para otro tipo de aguas. Por ese motivo, tanto las empresas como hospitales deben obtener su tratamiento primario. "Los hospitales e industrias no hacen tratamientos primarios de sus aguas", recalcó.

El tratamiento primario consiste en hacer una planta pequeña interna que tenga separadores de diferentes tipos de residuos, que necesariamente debe contar con esos filtros; una vez que esta agua pase por los filtros, recién sale solamente el agua al alcantarillado y esta podrá ser tratada en las lagunas de oxidación.

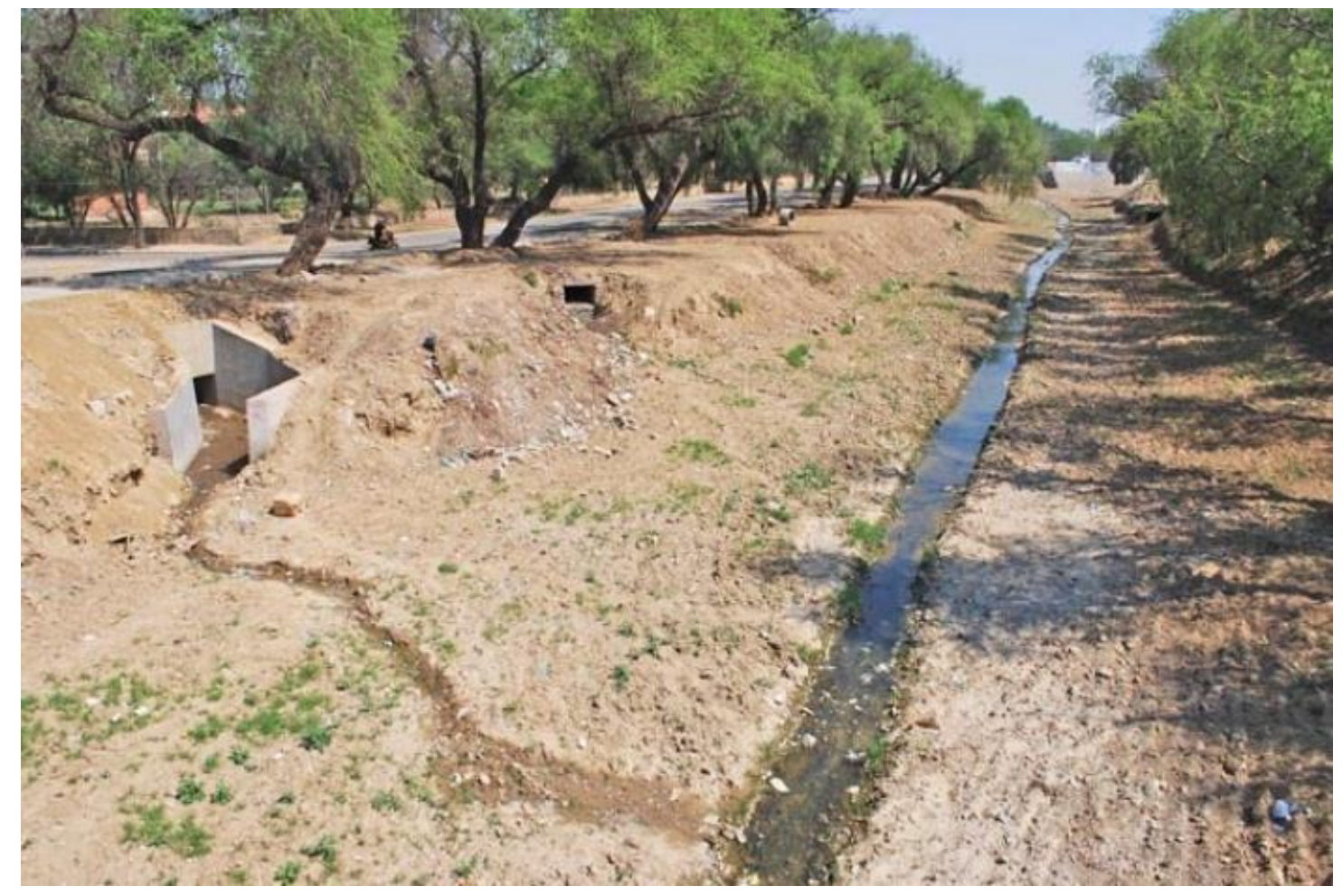

Figura 1. Quebrada EL Monte de la ciudad de Tarija Fuente: Gutiérrez, (El País, Oct 18, 2014) http://www.elpaisonline.com/index.php/2013-01-15-14-16-26/centrales/item/144021-hayquejas-por-basura-y-malos-olores-de-la-quebrada-el- monte
Levantamiento topográfico de la zona de estudio
Al momento de iniciar el punto ubicado en las inmediaciones del antamiento topográfico se realizó la Coliseo Universitario perteneciente a la U.A.J.M.S. levantamiento topográfico se realizó la
georreferenciación mediante un BM 
Tabla 2. Coordenadas del banco de nivel de precisión.

\begin{tabular}{llll}
\hline PUNTO & ESTE & NORTE & COTA \\
\hline BM & 321926.206 & 7616813.748 & 1863.323 \\
\hline
\end{tabular}

Posteriormente con la ayuda de una brújula se orientamos hacia el norte y procedimos a realizar el levantamiento topográfico a lo largo del tramo de estudio, dicha etapa llevo a cabo con la utilización de la estación total GEOMAX equipo que fue de gran ayuda gracias a su fácil manejo y precisión en sus datos (Tabla 2). Además, se usó el programa Civil - 3D para la extracción de las secciones transversales a ser ingresadas en el HEC-RAS.

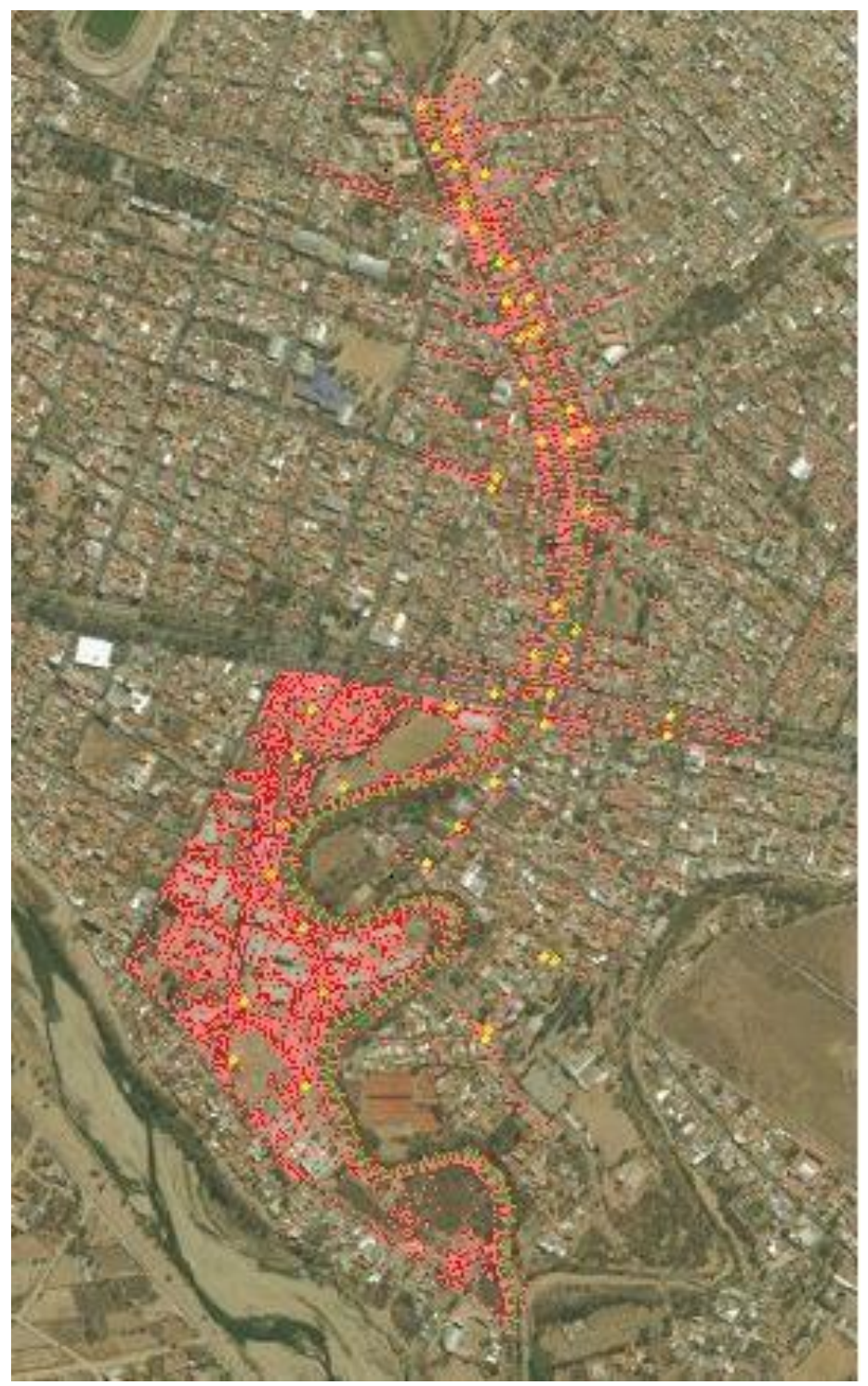

Figura 2. Croquis de la topografía en el tramo de estudio de la Quebrada EL Monte. 
Una vez introducidos los puntos al Civil-3D se procedió a un análisis exhaustivo de los datos para generar un modelo lo más aproximado posible a la realidad; posteriormente se generó la superficie del terreno, alineamiento ubicado en el eje de la Quebrada y finalmente la ubicación de las secciones a lo largo de todo el tramo de estudio previo análisis de las mismas. (Figura 2).

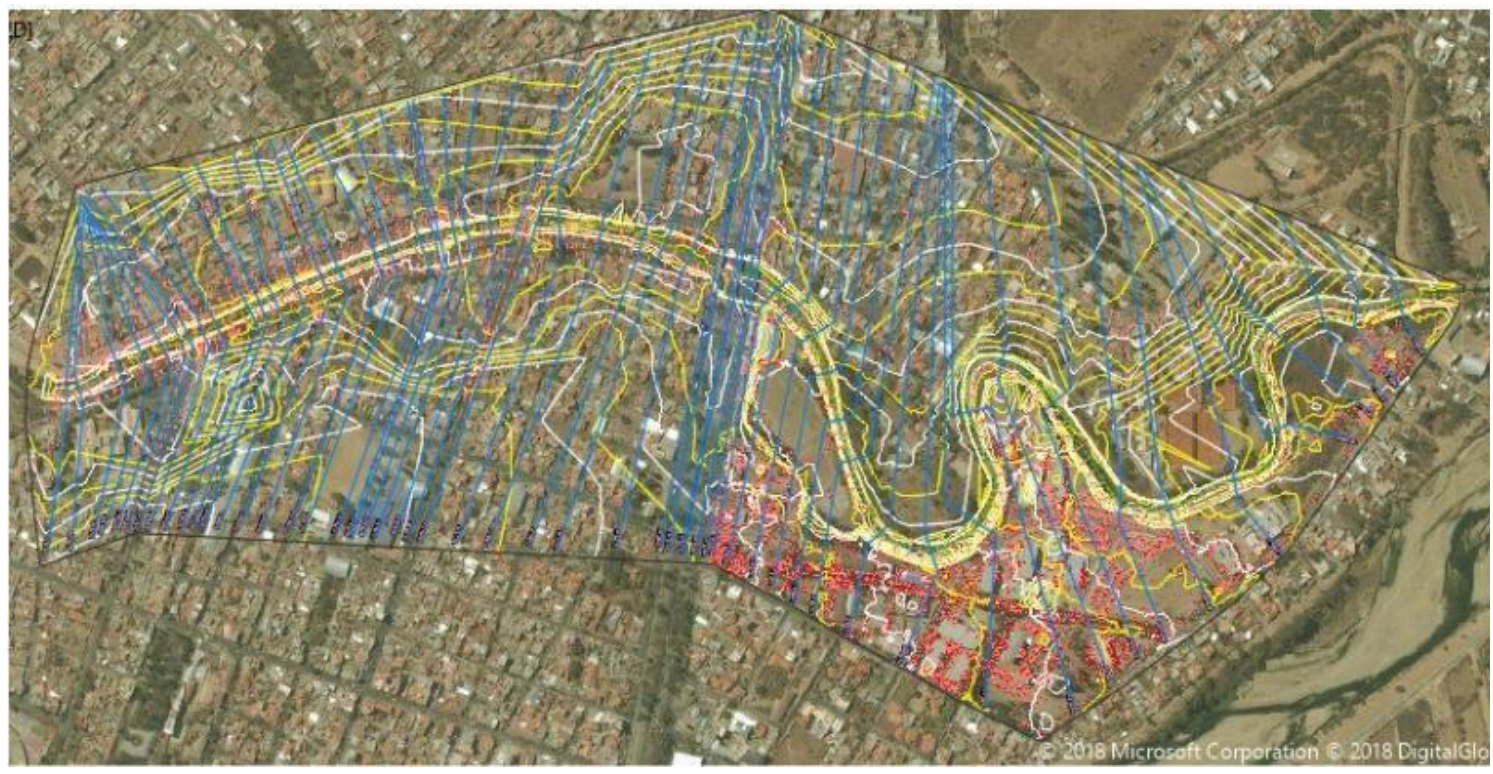

Figura 3. Superficie de terreno en Civil 3D. Fuente: Sánchez y Cardozo 2018.

\section{Exportación de los datos generados en Civil-3D a HEC-RAS}

HEC-RAS es un paquete integrado de análisis hidráulico, programa en que el usuario actúa recíprocamente con el sistema a través del uso de una Interface Gráfica del Usuario (GUI). El sistema es capaz de realizar cálculos de los flujos de la superficie del agua, cálculos de flujo uniforme, e incluirá flujo variado, transporte de sedimentos, etc. En la terminología del HEC-RAS, un proyecto es un juego de archivos de datos asociados con el sistema de un río en particular. (Figura 3). El modelador puede realizar cualquiera o varios tipos de análisis, incluidos en el paquete de HEC-RAS, como parte del proyecto.
Los archivos de datos para un proyecto se categorizan como sigue:

- Datos de planta.

- Datos geométricos.

- Datos de flujo uniforme.

- Datos de flujo variado.

- Datos de sedimentos

- Datos de diseño hidráulico.

Al exportar los datos del Civil-3D al software HEC-RAS automáticamente se visualiza la geometría del río con las secciones transversales definidas previamente, con el componente del RAS $>$ Geometric data (Figura 4). 


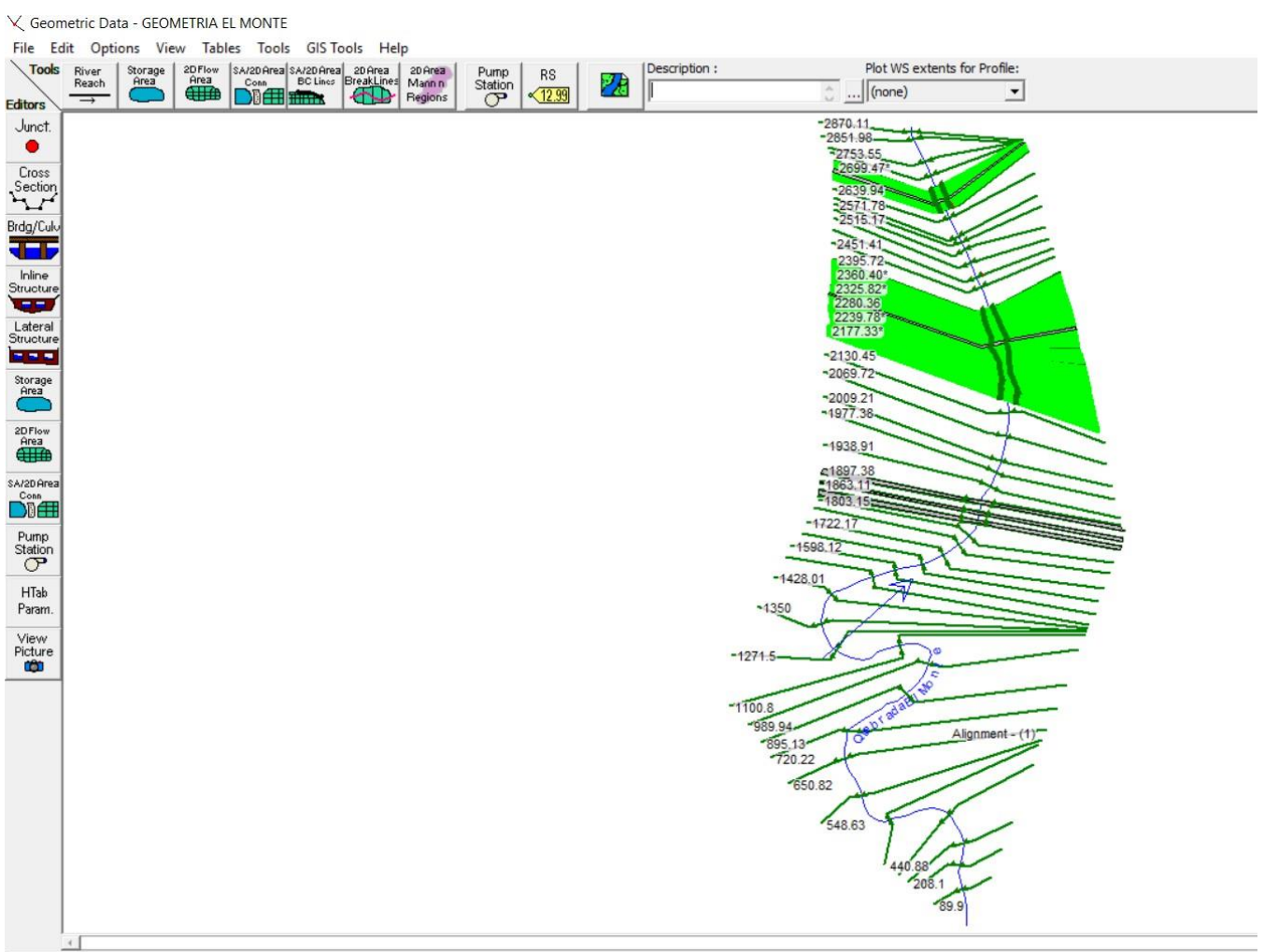

Figura 4. Geometría del río en HEC-RAS. Fuente: Sánchez y Cardozo 2018.

A continuación de obtener la el uso del suelo de acuerdo a los valores ya geometría del río se debe asignar a cada sección transversal el valor del coeficiente " $\mathrm{n}$ " de Manning; se estableció los valores en base a las condiciones naturales del cauce y determinados en el libro de "Hidráulica de Canales Abiertos" de Chow Te Ven, (2004) por tanto, se obtuvo valores para cada sección transversal.

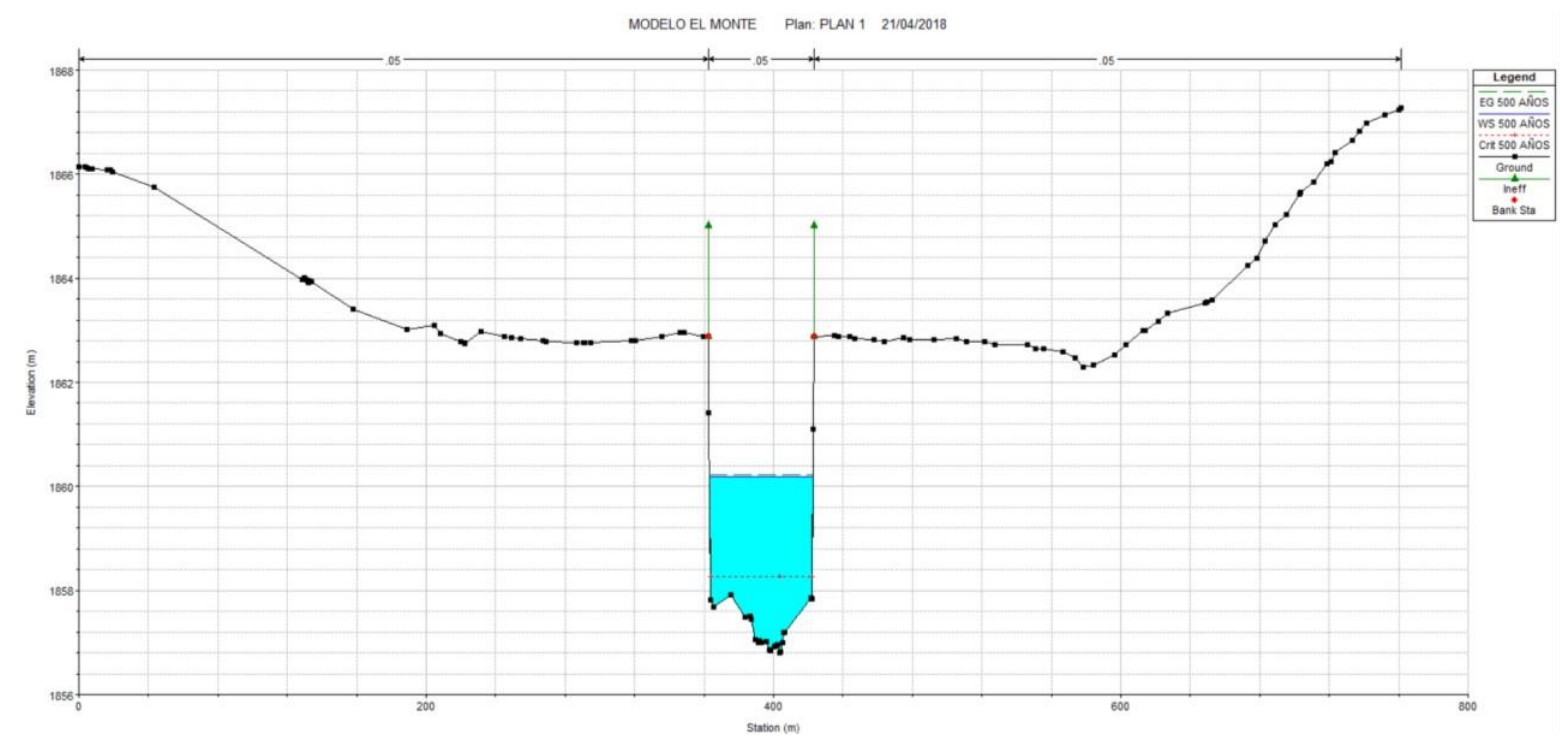

Figura 5. Ejemplo de sección transversal de la Quebrada El Monte. Fuente: Sánchez y Cardozo 2018. 
El ejemplo de sección transversal que se muestra en la Figura 5. Pertenece a la parte media del río, los puntos rojos delimitan el cauce principal del río (Banks Lines), la zona rellenada de celeste es la altura que ascenderá el agua para la simulación en diferentes períodos de retorno $(2,5,10,25,50,100,300,500$ años).

Luego se ingresar los valores de " $n$ " de Manning se debe introducir los caudales que se han obtenido mediante el software HMS para los diferentes periodos de retorno $(2,5,10,25,50,100,300,500$ años); debe completar las condiciones de contorno del canal (Boundary
Conditions) que son aquellas que definen el comportamiento del modelo en sus límites. HEC-RAS permite modelizar en régimen de flujo permanente $\mathrm{y}$ define hasta cuatro tipos distintos de condiciones de contorno: nivel de agua, profundidad crítica, curva de caudal y profundidad normal.

Para este estudio se usó la opción de profundidad crítica (Critical depth), ya que es la que más se acerca a los valores reales. Una vez especificado el tipo de condición de contorno se debe definir tanto para aguas arriba como aguas abajo (Figura 6).

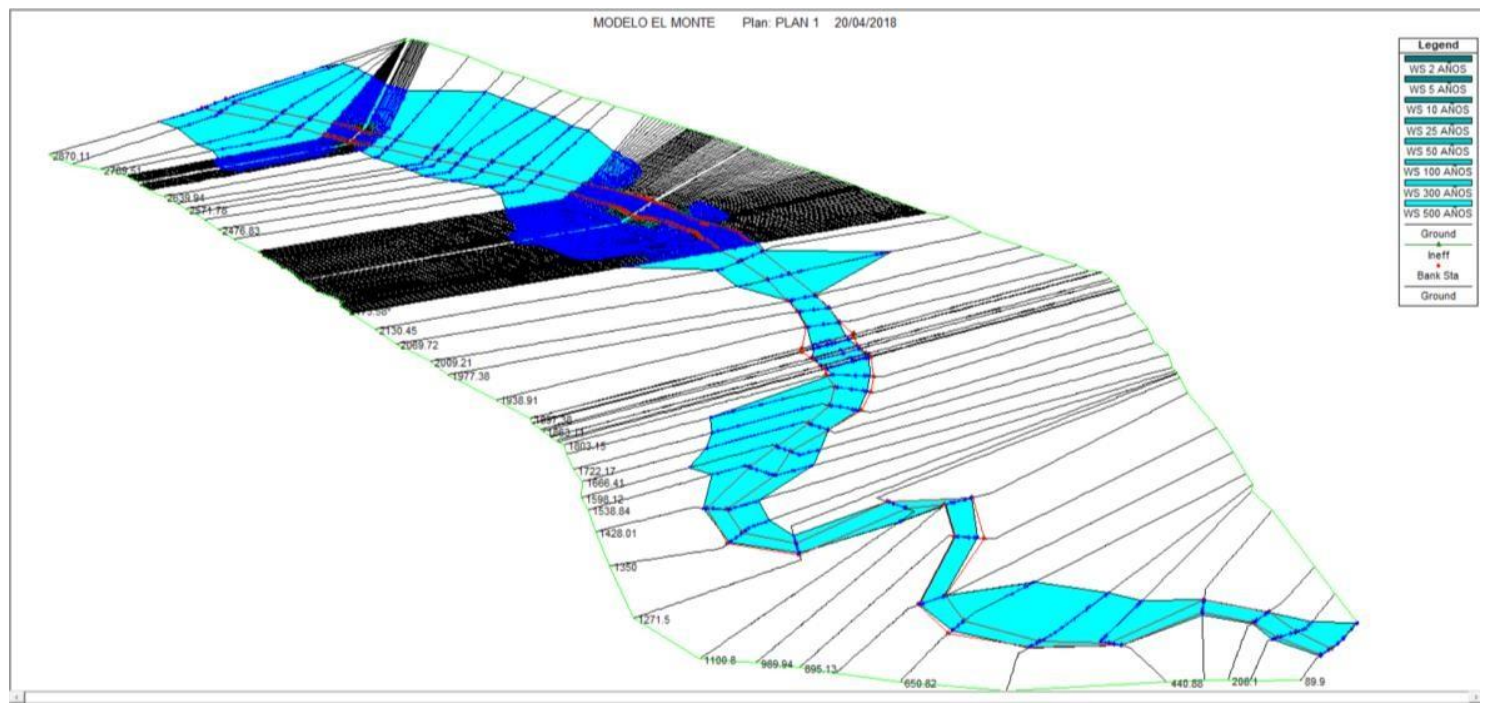

Figura 6. Simulación con HEC-RAS de la Quebrada El Monte. La zona en celeste representa la altura hasta dónde puede alcanzar el agua para periodo de retorno de 500 años. Fuente: Sánchez y Cardozo 2018. 


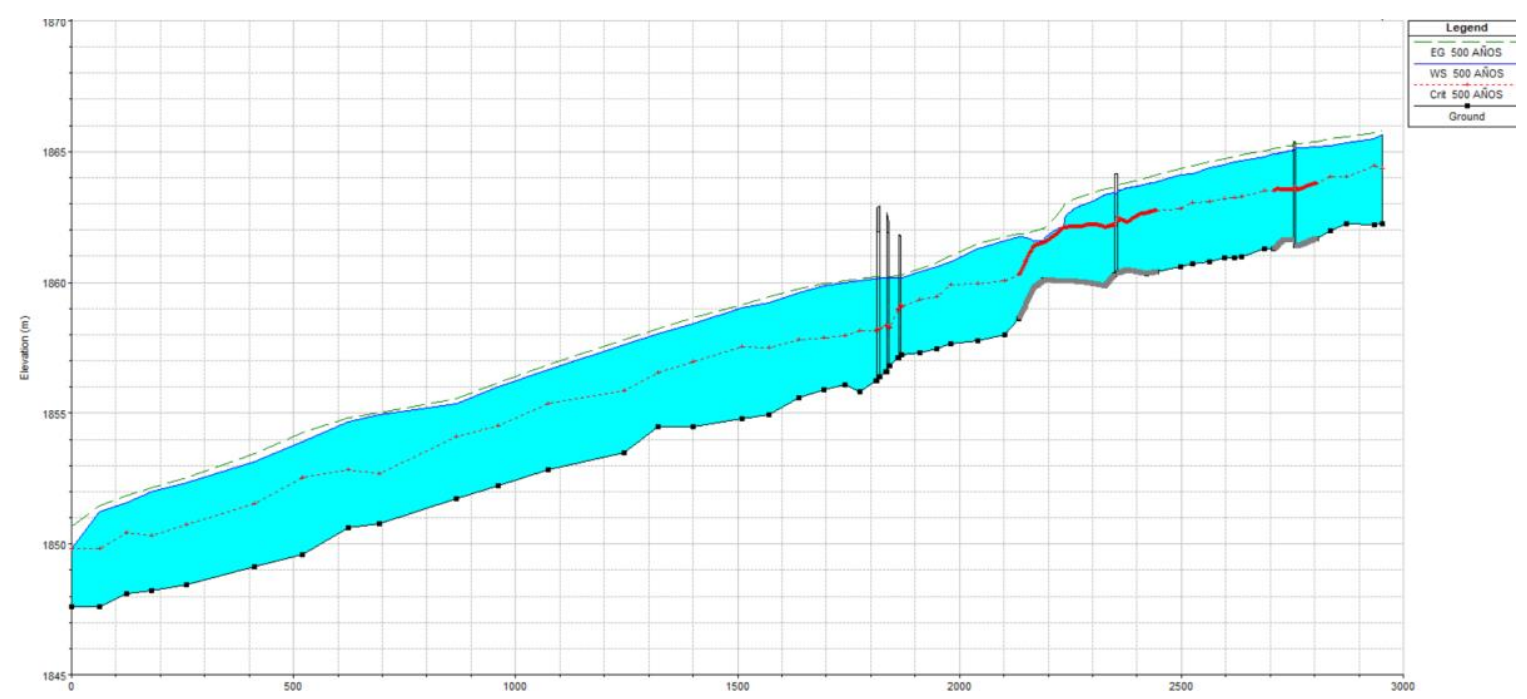

Figura 7. Perfil de la Quebrada El Monte. La zona en celeste representa la altura hasta dónde puede alcanzar el agua para periodo de retorno de 500 años. Fuente: Sánchez y Cardozo 2018.

\section{Resultados del análisis hidráulico REC-RAS}

Los valores obtenidos del análisis hidráulico mediante el programa HEC-RAS mediante la proyección del perfil de la Quebrada El Monte. (Figura 7).

\section{Discusión}

Una vez expuestos los resultados obtenidos en las sucesivas etapas que componen los análisis hidrológico e hidráulico, se procede a la discusión de los mismos. También se justificará los métodos empleados para cada análisis y los posibles errores (software) detectados a lo largo de la investigación.

\section{Análisis Hidráulico HEC-RAS}

Los resultados obtenidos de secciones tanto de los puentes y del terreno son representativos; sin embargo, la configuración de estos está constantemente sometida a cambios, incluso la sección del río es cambiante de manera permanente, por lo tanto, los resultados de tirantes máximos pueden ser diferentes todo el tiempo. Al tener configuraciones del río y del terreno en general todo el tiempo, el alcance de inundación puede ser variable a consecuencia del cambio de configuraciones del terreno.

La definición de diferentes valores para " $n$ " de la fórmula de Manning (tomados de bibliografía), por las condiciones de suelo y cobertura junto a las zonas de flujo infectivo, ha variado en las secciones con lo cual la velocidad es afectada directamente.

El estudio realizado en el Quebrada El Monte, desde el punto de vista de su comportamiento hidráulico, fue suficiente para permitir el esbozo de una solución al problema de las inundaciones.

La situación actual de la Quebrada El Monte es crítica, los problemas que presenta se los puede enumerar de la siguiente manera: 
- Basura que aumenta día a día lo cual reduce el área de las secciones transversales de la quebrada.

- El acelerado fenómeno erosivo característico de las cuencas de esta zona, porque la pérdida de la capa de suelo reduce el efecto típicamente retardador de la vegetación, contribuye al incremento de la parte de la lluvia que escurre, así como a aumentar la velocidad de las corrientes.

- La complejidad del tránsito de las crecidas a través del cauce estudiado difícilmente puede aproximarse por métodos convencionales que suponen régimen permanente uniforme.

\section{Consideraciones finales}

La basura y los matorrales que existen a lo largo del tramo de estudio generan una elevación del tirante a lo largo de toda la Quebrada. Además, Los gaviones construidos en las inmediaciones del Hospital Obrero ayudan a evitar erosiones, transporte de materiales y derrumbamientos de márgenes, además de controlar crecientes protegiendo la infraestructura del mismo contra inundaciones.

Para lo que una vez concluido el levantamiento topográfico de la zona de estudio se procedió a un exhaustivo análisis de datos, llegando a obtener un modelo real de la zona estudiada. Realizando así, la modelación hidráulica donde se observó que para los caudales de los primeros años de periodo de retorno las secciones presentan inundación no por desbordamiento sino debido a que en ambos extremos de la quebrada las cotas de terreno son más bajas que las cotas de los bordes de la quebrada.

El puente ubicado a la altura de la avenida Defensores del Chaco, al poseer accesos tan pronunciados genera que las secciones próximas a las mismas sean propensas al estancamiento de aguas.

Para la simulación del caudal de diseño que corresponde a un periodo de retorno de 500 años se observó que en las secciones de los puentes el tirante máximo no sobrepasa la cota mínima del tablero.

Para la modelación no se realizó el análisis de transporte de sedimentos.

El tramo ubicado a la altura de la avenida Belgrano presenta un número de Froude igual a 2.1, esto debido a que en esta zona se encuentra un badén que funciona como un vertedero cuya función en la disipación de energía aguas abajo.

En al tramo del barrio San Gerónimo las áreas propensas a inundación podrían ser mayores, esto es debido a que existen dos cauces naturales de gran importancia (Río Guadalquivir y Quebrada San Pedro) en las proximidades y sus áreas de desbordamiento pueden llegar a afectar a la zona mencionada.

\section{CONCLUSIONES}

Se puede apreciar que tanto el modelo hidrológico como el modelo hidráulico para la Quebrada el Monte son correctos, esto mediante observación de eventos pasados de inundación, los cuales corroboran los datos obtenidos.

Se concluye lo siguiente:

- Zona Hospital Obrero: se ve afectada en los periodos de retorno de $25,50,100$, 300 y 500 años específicamente entre las secciones 2789.51 y 2720.91 .

- Zona Cine La Torre: se ve afectada en los periodos de retorno de $10,25,50$, 
100, 300 y 500 años entre las secciones 2451.41 y 2009.21, debido que presenta una zona de baja profundidad al existir obras civiles en este caso el puente de la Av. Defensores del Chaco.

- Zona Coliseo Universitario: se ve afectada en los periodos de retorno de $50,100,300$ y 500 años entre las secciones 1939.3 y 1538.84 .

- Zona Tarija Tenis Club: se ve afectada en los periodos de retorno de 10, 25, $50,100,300$ y 500 años entre las secciones 720.22 y 287.04 , es una zona con altura de desbordamiento provisional debido a la unión de la Quebrada San Pedro lo cual generaría un incremento en la mancha de inundación para esta zona.

- Los gaviones que existen en la quebrada El Monte en el tramo de estudio son simplemente de protección de laderas, por lo que resulta insuficiente para el riesgo de inundación existente en la zona.

Por otra parte se recomienda que:

Se recomienda instalar más Estaciones Pluviométricas para mayor exactitud en el modelo hidrológico e hidráulico.

Implementar un adecuado uso y manejo de los suelos, orientado a prevenir los efectos de la erosión hídrica y controlar el volumen y velocidad del agua de escorrentía, unidos a campañas de reforestación de las riberas de los ríos con bosques de galería para el control de las corrientes.

Establecimiento de obras hidráulicas en aquellos puntos críticos que presentan problema de drenajes, con el fin de darle solución a la problemática de accesibilidad a algunas áreas productivas del municipio y de tránsito poblacional.
Se puede implementar una franja de seguridad aledaña a la Quebrada El Monte para evitar futuras construcciones $\mathrm{y}$ asentamientos, así también para ampliar el Macro proyecto de la cuenca del Río Guadalquivir con el desarrollo del potencial de Inundación de la Quebrada San Pedro para así tener más detalles de la Zona de Tarija Tenis Club (Zona con altura de desbordamiento provisional).

Orientar a la población mediante campañas de concientización sobre la importancia de evitar el vertido de residuos sólidos u otras sustancias contaminantes a la Quebrada el Monte en el tramo de estudio.

\section{REFERENCIAS}

Chow Ven Te, "Hidráulica de Canales Abiertos" editora, Martha Edna Suarez R. Colombia, 2004

El Nacional (nov 10 2010). Las quebradas se convirtieron en basureros y cementerios e chatarra. Disponible en: http://www.elnacionaltarija.com/diari o/index.php/2010/11/10/lasquebradas-se- convirtieron-enbasureros-y-cementerios-de-chatarra/ (Publicado en 10 noviembre 2010) Bolivia

Gutiérrez, W., P. (El País, Oct 18, 2014). Hay quejas por basura y malos olores de la quebrada El Monte. Disponible en: http://www.elpaisonline.com/index.p hp/2013-01-15-14-16-

26/centrales/item/144021- hayquejas-por-basura-y-malos-olores-dela-quebrada-el-monte

Olaguivel Quiroga, C, (1999). Estudio de Riesgo de Inundación de la Quebrada de San Pedro". Trabajo de Tesis para optar por el Grado de Licenciado en Ingeniería Civil U.A.J.M.S. Tarija

Programa Estratégico de Acción, PEA. (1999). Control de Inundaciones de la Ciudad de Tarija 
Ribstein, P, y Pena, J (1993 citado por AA 2014). Estudio hidrológico para la protección contra las inundaciones de Villamontes (Bolivia)

Terrazas G., AR, (2011). Evaluación del Riesgo de Inundación de la Quebrada
El Monte de la ciudad de Tarija. Trabajo de Tesis para optar por el Grado de Licenciado en Ingeniería Civil U.A.J.M.S. Tarija 